\title{
Optimization of temperature-programmed gas chromatographic separations, II: Off-line Simplex optimization and column selection
}

Citation for published version (APA):

Snijders, H. M. J., Janssen, J. G. M., \& Cramers, C. A. M. G. (1996). Optimization of temperature-programmed gas chromatographic separations, II: Off-line Simplex optimization and column selection. Journal of Chromatography, A, 756(1-2), 175-183. https://doi.org/10.1016/S0021-9673(96)00626-7

DOI:

10.1016/S0021-9673(96)00626-7

Document status and date:

Published: 01/01/1996

Document Version:

Publisher's PDF, also known as Version of Record (includes final page, issue and volume numbers)

Please check the document version of this publication:

- A submitted manuscript is the version of the article upon submission and before peer-review. There can be important differences between the submitted version and the official published version of record. People interested in the research are advised to contact the author for the final version of the publication, or visit the $\mathrm{DOI}$ to the publisher's website.

- The final author version and the galley proof are versions of the publication after peer review.

- The final published version features the final layout of the paper including the volume, issue and page numbers.

Link to publication

\footnotetext{
General rights

- You may freely distribute the URL identifying the publication in the public portal. follow below link for the End User Agreement:

www.tue.nl/taverne

Take down policy

If you believe that this document breaches copyright please contact us at:

openaccess@tue.nl

providing details and we will investigate your claim.
}

Copyright and moral rights for the publications made accessible in the public portal are retained by the authors and/or other copyright owners and it is a condition of accessing publications that users recognise and abide by the legal requirements associated with these rights.

- Users may download and print one copy of any publication from the public portal for the purpose of private study or research.

- You may not further distribute the material or use it for any profit-making activity or commercial gain

If the publication is distributed under the terms of Article $25 \mathrm{fa}$ of the Dutch Copyright Act, indicated by the "Taverne" license above, please 


\title{
Optimization of temperature-programmed gas chromatographic separations \\ II. Off-line Simplex optimization and column selection
}

\author{
Henri Snijders*, Hans-Gerd Janssen, Carel Cramers \\ Laboratory of Instrumental Analysis, Department of Chemical Engineering. Eindhoven University of Technology, P.O. Box 51.3. 5600 \\ $M B$ Eindhoven, Netherlands
}

Received 3 May 1996; revised 9 July 1996; accepted 15 July 1996

\begin{abstract}
In this work a method is described which allows off-line optimization of temperature-programmed GC separations. The method is based on a new numerical method that allows the off-line prediction of retention times and peak widths of a mixture containing components with known identities in capillary GC. In the present work we apply this method for off-line optimization of single- and multi-ramp temperature-programmed GC separations. First, it will be shown how the numerical methods are incorporated in a Simplex optimization method. Next, it is described how the method can be used to determine the optimal temperature program for the separation of a mixture containing components of different functionalities. Finally, it is shown that the optimization strategy followed here allows selection of the capillary column most suited for the separation problem under study from a given set of capillary columns containing the same stationary phase and varying inner diameters and film thicknesses. The process can be performed without any experimental effort. The results indicate that fully off-line simulation and optimization of single- and multi-ramp temperature-programmed GC separations as well as column selection is possible.
\end{abstract}

Keywords: Simplex optimization; Temperature-programming; Optimization; Column selection; Capillary columns; Chemometrics

\section{Introduction}

In a previous article [1] we described a numerical method to predict (truly off-line) linear temperatureprogrammed retention times and peak widths for mixtures containing components with known identities in capillary GC. The procedure is based on

* Corresponding author. extracting thermodynamic values (entropy and enthalpy terms) from published Kováts retention indices. A numerical method was developed, that uses these thermodynamic values to calculate linear (single or multi-ramp) temperature-programmed retention times and peak widths on any capillary GC column containing the same stationary phase but with a different phase ratio and/or length. In short, the numerical approach models the solute's chromatographic process into very small segments of equal time. For every time interval the various 
chromatographic parameters are calculated, based on a model developed from sound chromatographic theory. Retention times are calculated from local retention factors and the pressure and temperature corrected local velocity in every time interval. Analogously, peak widths are calculated from local plate heights (derived from the well-known Golay equation) in every segment. During the calculations physical models and approximations are used to obtain viscosity and diffusion data.

Apart from the thermodynamic quantities and the solutes molecular formula, the only additional input parameters needed for the predictions are the column characteristics (dimensions and coating efficiency), the carrier gas type and several instrumental parameters such as the temperature program and the column dead time or inlet pressure. In the temperature-programmed mode a wide temperature range is allowed and isothermal plateaus can be included in the program. An attractive feature of the approach is that, once the thermodynamic values of the solutes of interest are known, they can be stored in a database and future predictions can be performed without the need to perform new experimental input runs.

In the present work we apply this new numerical method to off-line optimization of temperature-programmed GC separations, where we define the term optimization as achieving baseline resolution in the shortest possible analysis time. The ultimate goal of the work is to develop an optimization strategy that does not require any experimental input run. In other words, a method that enables truly off-line optimization. First, it will be shown how the numerical methods for retention time and peak width prediction previously developed are incorporated in a Simplex optimization method. Next, it is described how the method can be used to determine off-line the optimal (either single- or multi-ramp) temperature program for the separation of a mixture containing components of different functionalities. Finally, it is shown that the optimization strategy followed here allows selection of the capillary column most suited for the separation problem under study, from a given set of capillary columns containing the same stationary phase but with varying phase ratios and/or lengths.

\section{Theory}

\subsection{Chromatographic response function}

Successful application of an optimization process is preceded by careful selection of the quantity (response factor) to be optimized. For chromatographic separations the main problem lies in proper conversion of the retention times and peak widths of all relevant chromatographic peaks into a single number which accurately reflects the optimization criterium. Several authors have recognized and discussed this problem [2-4] and many chromatographic response functions (CRF) have been suggested. In this work we adopted the CRF used by Dose [5]. For a given chromatogram the CRF value can be calculated from:

$\mathrm{CRF}=\frac{t_{\mathrm{R} n}}{t_{\mathrm{R}, \mathrm{crit}}}+\sum_{i \neq j} e^{-\frac{R_{\mathrm{s}, i j}}{R_{\mathrm{s}, \mathrm{rrit}}}}$

where $t_{\mathrm{R}, n}$ is the retention time of the last-eluting peak, $t_{\mathrm{R}, \text { crit }}$ is a user-selected time-cost weighting or compromise factor and $R_{\text {, crii }}$ is a user-selected resolution target value. $R_{\mathrm{s}, i j}$ is the resolution between peak $i$ and $j$ which is defined as:

$R_{\mathrm{s}, i j}=\frac{\Delta t_{\mathrm{R} . i j}}{2\left(\sigma_{i}+\sigma_{j}\right)}$

where $\Delta t_{\mathrm{R}, i j}$ is the retention time difference (expressed positive) between peak $i$ and $j . \sigma_{i}$ and $\sigma_{i}$ are the standard deviations of peak $i$ and peak $j$, respectively.

From Eq. (1) it can be seen that both analysis time and resolution are incorporated in the CRF. Moreover, all possible peak-to-peak interactions are included. The occurrence of overlapping peaks is strongly discouraged. Additionally, it emphasizes the least resolved peak pairs without totally ignoring the other peak pairs. Further merits of the CRF used here are extensively discussed by Dose [5].

\subsection{Optimization algorithm}

The optimization procedure used in this work is the Modified Simplex Method (MSM) as described 
by Nelder and Mead [6]. Successful application of the Simplex method for the optimization of GC separation problems was demonstrated by several authors, e.g. [5,7]. In this section it will be shown how the numerical methods previously derived for the calculation of retention times and peak widths in temperature-programmed GC separations [1] are incorporated in an MSM for the purpose of off-line optimization of temperature-programmed GC separations. Moreover, it will be shown how the strategy followed here allows selection of the capillary column most suited for a given separation problem from a set of available columns. The discussion of the various important aspects of the optimization process is guided by the overall optimization algorithm, presented in Fig. 1.

Prior to starting the optimization, it must be decided which parameters are to be optimized. Normally only those factors are included which exhibit the largest impact on the CRF (the quantity to be optimized). For the majority of GC separations the temperature-programming rate is the decisive parameter in order to attain the ultimate separation goal. Apart from the programming rate, also other important parameters such as e.g. the initial oven temperature, can be included in the optimization. With the present algorithm the number of dimensions to be optimized can be fully user-selected. This means that the optimization can be performed for single- and/or multi-ramp temperature-programmed separations either with or without the incorporation of isothermal plateaus. In fact, all variables which describe a GC temperature program can be included as optimization parameters. Parameters which are not included in the optimization are held constant.

As already mentioned in the introduction, the primary goal of the work presented here is to attain truly off-line optimizations. During the optimization calculations the mean linear velocity at the initial oven temperature was kept constant. Hence, the numerical methods applied here require a representative value for the mean linear velocity. This value was estimated for a given combination of column inner diameter and carrier gas type using Table 1. This table is compiled by using the computer program described in [8]. With the value from the table and given the initial oven temperature (determined

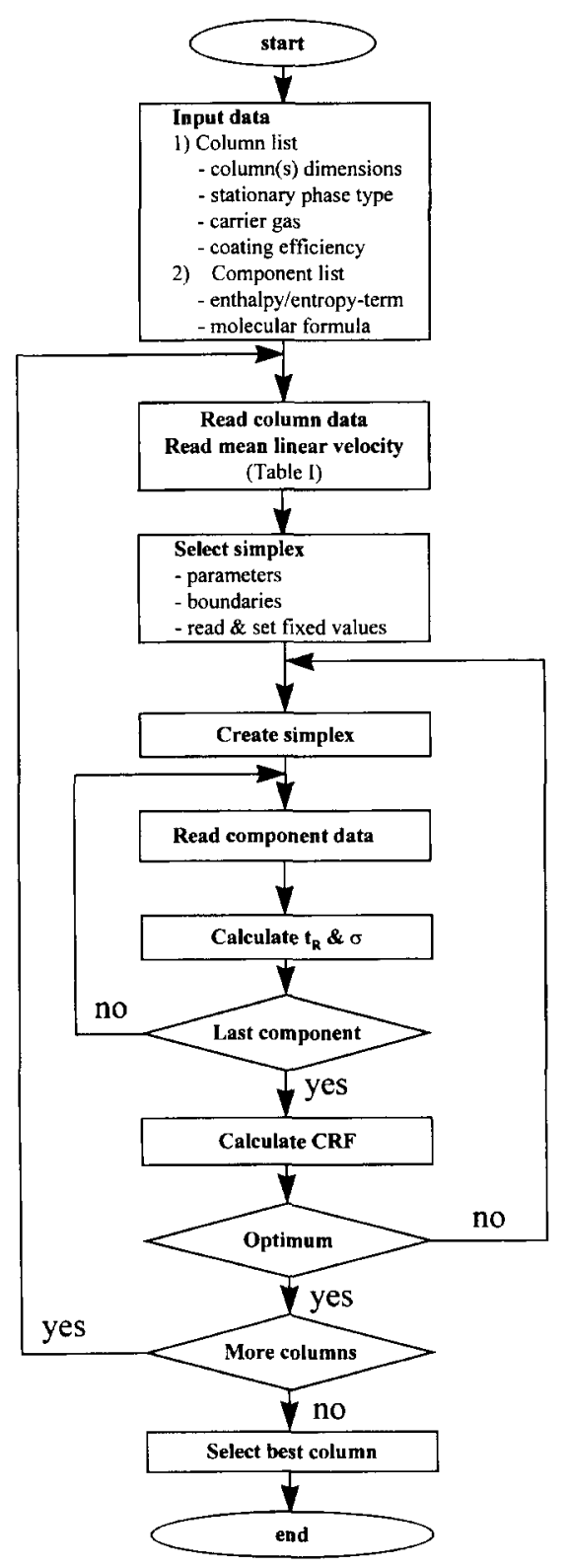

Fig. 1. Overall algorithm of the optimization procedure.

by the vertex data), the corresponding column dead time and required inlet pressure can be calculated using the approach described in part I of this work [1]. This means that during the execution of the optimization no experimental data have to be ac- 
Table 1

Estimated mean linear velocities [8] as a function of column diameter and carrier gas type

\begin{tabular}{llll}
\hline Column inner diameter $(\mu \mathrm{m})$ & \multicolumn{3}{l}{ Mean linear velocity $\left(\mathrm{cm} \mathrm{s}^{-1}\right)$} \\
\cline { 2 - 4 } & \multicolumn{3}{l}{ Carrier gas type } \\
\cline { 2 - 4 } & $\mathrm{H}_{2}$ & $\mathrm{He}$ & $\mathrm{N}_{2}$ \\
\hline $150-320$ & $55-45$ & $40-33$ & $17-12$ \\
$321-550$ & $45-28$ & $33-25$ & $12-7$ \\
\hline
\end{tabular}

The values for the mean linear velocities are retrieved from the table by linear interpolation between the indicated column diameters.

quired, which satisfies our goal of truly off-line optimization.

An other important aspect to be addressed are the constraints which have to be put on the variables to be optimized. Often, those boundaries are dictated by the chromatographic equipment used. As an example, contemporary gas chromatographs have limited maximum programming rates. Moreover, the maximum and minimum allowable operating temperatures of the separation column are limited. Also parameter values that would lead to highly undesirable results such as too low a programming rate or too low an initial oven temperature can be excluded from the factor space. By taking those limitations into account, the factor space can thus be adapted to practical and/or experimental considerations. The simplex is held within the boundaries by assigning unfavourable (very high) CRF values to vertices which fall outside the factor space.

The location of the initial simplex should be carefully chosen. The classical problem associated with Simplex optimizations is that the final optimum may not be the global optimum but a local optimum with a less desirable response (in this case a less desirable CRF value). For GC optimizations convergence to a local optimum can easily occur when e.g. peak orders change under temperature-programmed conditions. To locate the optimum which yields the shortest possible analysis time (while maintaining baseline resolution), the initial simplex is selected near the upper or lower boundaries of the factor space. In case of a three-factor optimization this could mean that the initial simplex is located close to the maximum allowable programming rate and initial temperature and close to the minimum allowable time for an isothermal plateau.

Once the initial simplex is determined, the optimization procedure proceeds as follows. Given the conditions specified by the factors of each vertex of the initial simplex, the retention times and peak widths for each component are calculated by using the numerical procedures. For this purpose the entropy and enthalpy term and the molecular formula for each component must be known. The calculated peak data are than used to calculate the CRF for each vertex. The optimization continues by rejecting the vertex with the least desirable response followed by determination of the next simplex, guided by the Modified Simplex algorithm (note that a high CRF value indicates a low response!). The process is ended when the step size of each factor to be optimized is less than a preselected maximum allowable difference. This value is usually given by the accuracy of the instrumentation for each individual factor.

The process described above is restarted when the optimal chromatogram should be predicted for another column, with a different phase ratio and/or length, until all column data are processed. Finally, comparison of the results yields the column exhibiting the lowest analysis time (indicated by the retention time of the last-eluting component) for the given separation problem. Here it should be realized that in practical situations the final selection of the column most suited for the separation problem under study can also be guided by additional practical considerations such as e.g. the oven cooling down time.

\section{Experimental}

\subsection{Instrumentation}

GC was performed on an HP 5890 gas chromatograph equipped with a split-splitless injector and a flame ionization detection (FID) system (HewlettPackard, Wilmington, DE, USA). The columns used in this study are indicated in Table 2. Columns A, B and $\mathrm{D}$ are coated with $100 \%$ methyl silicone, HP-1 (Hewlett-Packard). Column C is coated with $100 \%$ methyl silicone, CP-Sil 5 CB (Chrompack, Middel- 
Table 2

Dimensions of the columns used in this study

\begin{tabular}{lllll}
\hline Symbol & $L(\mathrm{~m})$ & $d_{c}(\mu \mathrm{m})$ & $d_{\mathrm{r}}(\mu \mathrm{m})$ & $\beta$ \\
\hline A & 25 & 320 & 0.52 & 153 \\
B & 25 & 320 & 0.17 & 470 \\
C & 25 & 250 & 0.25 & 250 \\
D & 25 & 200 & 0.33 & 264 \\
\hline
\end{tabular}

$L=$ column length; $d_{\mathrm{c}}=$ column diameter; $d_{1}=$ stationary phase film thickness; $\beta=$ column phase ratio. All columns are coated with $100 \%$ methyl silicone.

burg, The Netherlands). Injections were performed in the split mode (split ratio 1:100) to minimize injection band broadening. The instrument was operated in the constant pressure mode. In experimental verifications of predicted optimal chromatograms, the optimal column dead time, reported after the optimization, was set on the instrument by adjusting the carrier gas (helium) pressure to the corresponding value via methane injections. Both injector and detector temperature were held constant at $300^{\circ} \mathrm{C}$ during the experimental work. The detector make-up gas flow-rate (nitrogen) was maintained at $30 \mathrm{ml}$ $\mathrm{min}^{-1}$. An Omega data system (Perkin-Elmer, Norwalk, CT, USA) was used for data acquisition and processing.

\subsection{Calculations}

During the simulations no correction for the column coating efficiency was applied. For the calculations it was assumed that the column outlet pressure equals $100 \mathrm{kPa}$ (abs.). The stepwidth $\Delta t$ was $1000 \mathrm{~ms}$ [1]. The maximum allowable difference for the factors is $1^{\circ} \mathrm{C}$ for temperatures and $0.1{ }^{\circ} \mathrm{C} \mathrm{min}{ }^{-1}$ for temperature programming rates. For CRF calculations, $R_{\text {s.crit }}$ and $t_{\mathrm{R} . \text { crit }}$ are set to 1.5 and $20 \mathrm{~min}$ respectively. All computations were carried out on a 486-DX2/66 MHz personal computer. Software was written in Turbo Pascal 6.0 (Borland, USA). A complete optimization for one column takes about 15 min. Data entry is arranged through filed input.

\subsection{Test mixture}

To determine the applicability of the optimization approach presented above, a test mixture was com- piled, containing sixteen components of different functionality. $p$-Chlorotoluene, sec-butylbenzene, diphenyl ether, anthracene, pyrene and hexadecene were purchased from Janssen Chimica (Geel, Belgium). Methyl esters of myristic acid, palmitic acid and oleic acid were obtained from Polyscience Corporation (IL, USA). $n$-Undecane, $n$-undecene, $n$ tridecane and $n$-tetradecane were purchased from Merck (Darmstadt, Germany). 1-Chlorotetradecane was obtained from Humphrey Wilkinson (CT, USA) and naphthalene and quinoline from Aldrich (Bornem, Belgium). The purity of all analytes was at least $98 \%$. The solvent used to prepare the mixture was analytical grade $n$-hexane (Merck).

The entropy and enthalpy terms of the test components were obtained from Ref. [1]. For test components for which no data were available the entropy and enthalpy terms were experimentally established.

\section{Results and discussion}

To demonstrate the applicability of the novel optimization approach, optimizations of the test mixture were performed for all columns. Ideally, the optimum located by the Simplex procedure should be the global optimum. To check whether the predicted optimum equals the global optimum, detailed knowledge of the response surface should be available. For a two-factor optimization the response surface can be graphically represented by e.g. a contour plot. In Fig. 2. the calculated response surfaces for the separation of the test mixture on all columns are presented. The two factors plotted are the initial column temperature and the oven programming rate. The CRF values were calculated off-line by using the numerical methods. In the contour plots the iso-response (iso$\mathrm{CRF}$ ) points are connected by lines. From the figure it can be seen that in all plots several local optima can be observed. This occurs e.g. when peak orders change during temperature programming. Closely located optima are observed when the gain in analysis time by starting the temperature program at a higher initial temperature is partially offset by a decrease in the programming rate. This leads to only minor differences in the chromatographic separation 
Column A

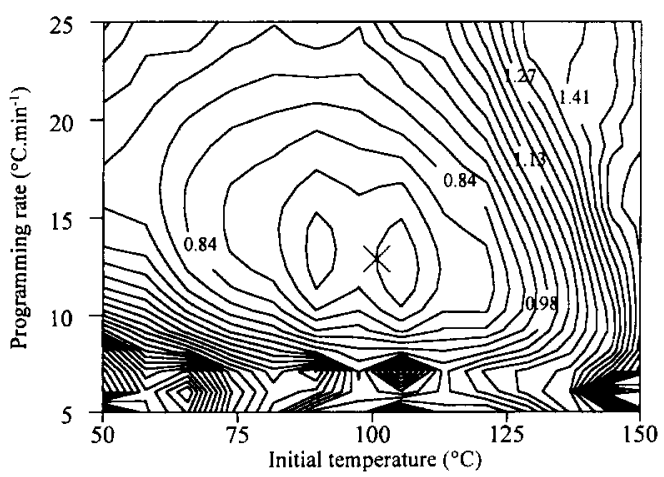

Column C

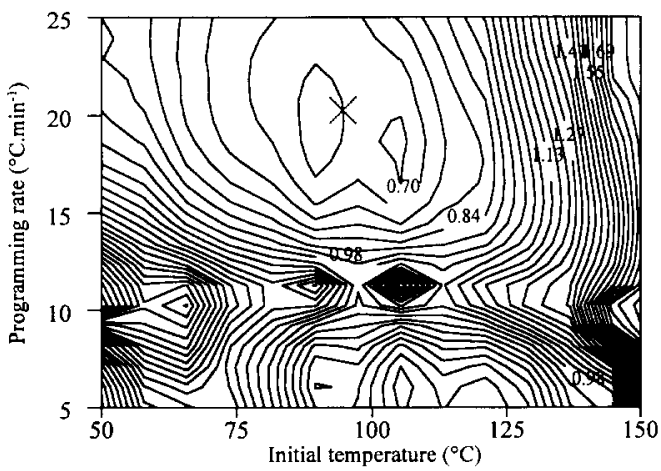

Column B

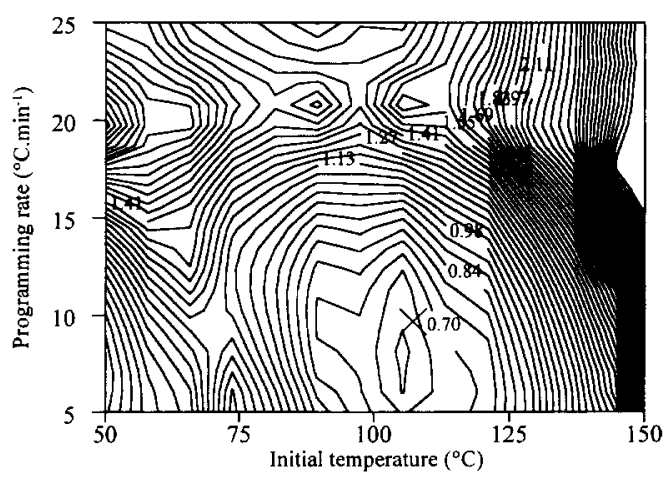

Column D

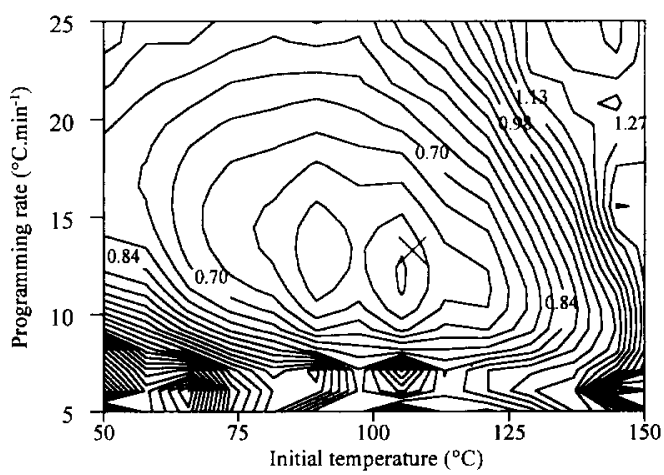

Fig. 2. Iso-response plots of the separation of the test mixture on all four the columns. Parameters included in the optimization are the initial oven temperature and the programming rate. The optima found by the corresponding Simplex optimization are indicated by the cross. The

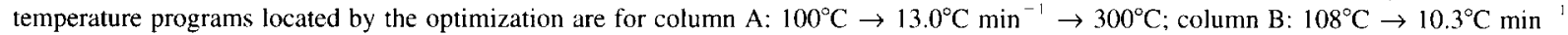
$\rightarrow 300^{\circ} \mathrm{C}$; column $\mathrm{C}: 97^{\circ} \mathrm{C} \rightarrow 20.4^{\circ} \mathrm{C} \min ^{-1} \rightarrow 300^{\circ} \mathrm{C}$; column $\mathrm{D}: 108^{\circ} \mathrm{C} \rightarrow 13.7^{\circ} \mathrm{C} \min ^{-1} \rightarrow 300^{\circ} \mathrm{C}$.

and/or analysis time. The corresponding CRF values are nearly equal.

In parallel, the corresponding two-factor Simplex optimization was performed for the test components for all columns. The parameters plotted in the contour plots were now optimized by using the novel optimization approach. No initial hold time was used. The final optima, located by the optimization, are indicated in the contour plots of Fig. 2 as well. From the figure it can be seen that the optima located in all cases equal the global optima.

To verify the resemblance between predicted and experimental optimal chromatograms, the test mix- ture was analyzed under the conditions determined by the values of the optimum for each column. The results of these measurements are presented in Fig. 3. The figure clearly shows that for all columns the experimental and predicted chromatograms are very similar. In the chromatograms several critical peak pairs can be distinguished. The experimental data corresponding to the optimization on column $\mathrm{C}$ and D reveal partial peak overlap for the second critical peak pair. Due to the smaller inner diameter of these columns, compared to columns $\mathrm{A}$ and $\mathrm{B}$, injection band broadening becomes more critical and is probably responsible for the partial peak overlap observed. 
Column A
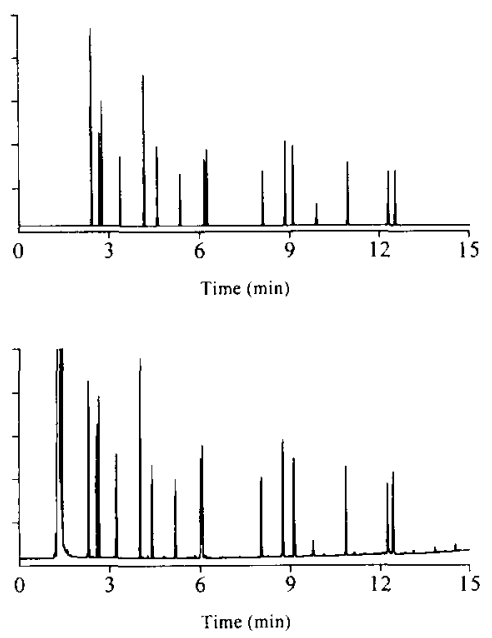

Column C
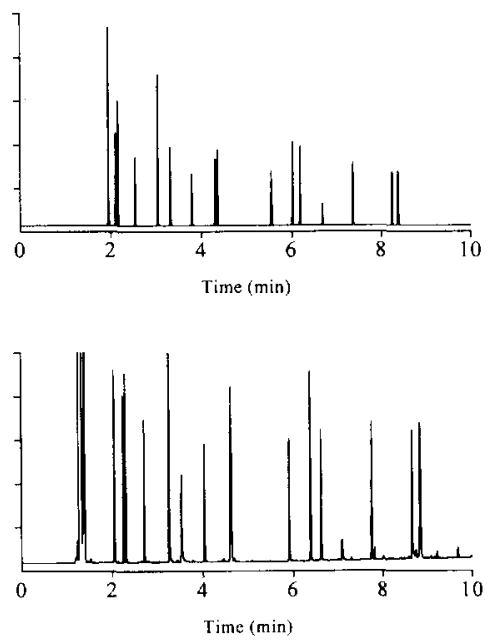

Column B
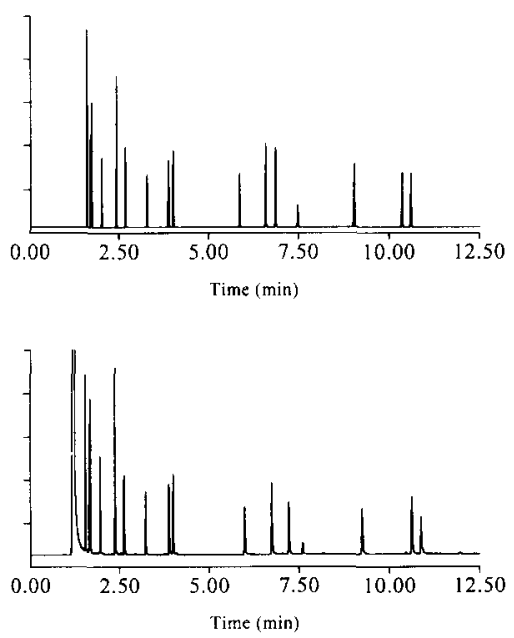

Column D
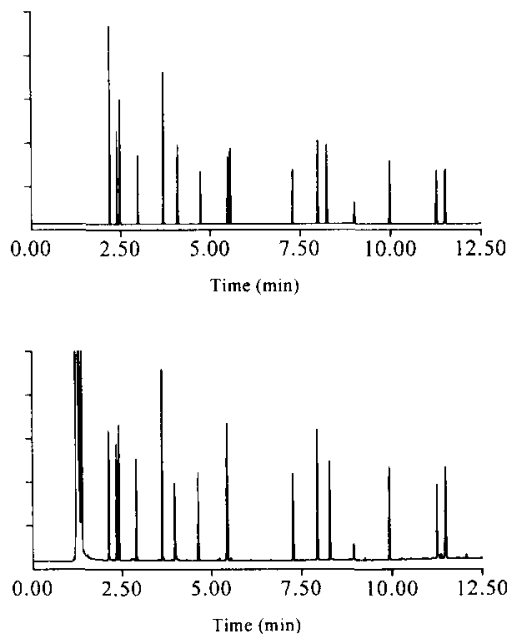

Fig. 3. Comparison of predicted (upper trace) and experimental (lower trace) chromatograms for single-ramp temperature-programmed optimizations of the separation of the test mixture for all four the columns. Conditions see Fig. 2.

The chromatographic data further indicate that the column most suited for single-ramp analysis of the mixture would be column $B$. This column yields the shortest analysis time while all critical peak pairs are baseline separated.

Optimization of the separation of the test mixture was also performed for a two-step multi-ramp temperature program. Parameters optimized now are the initial and mid-point column temperature and the corresponding two programming rates. In total four parameters are optimized simultaneously. Again, the predicted and experimental chromatograms are presented in Fig. 4. From the figure it can be concluded that for all four columns the experimental and predicted chromatograms are very similar. The experimental data corresponding to the optimizations 
Column A
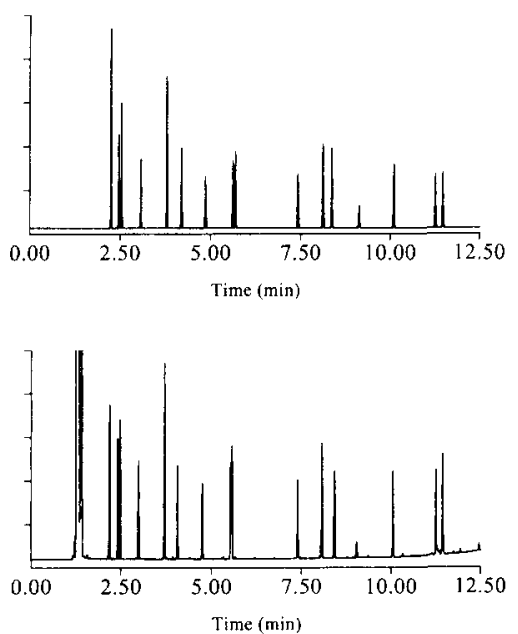

Column C
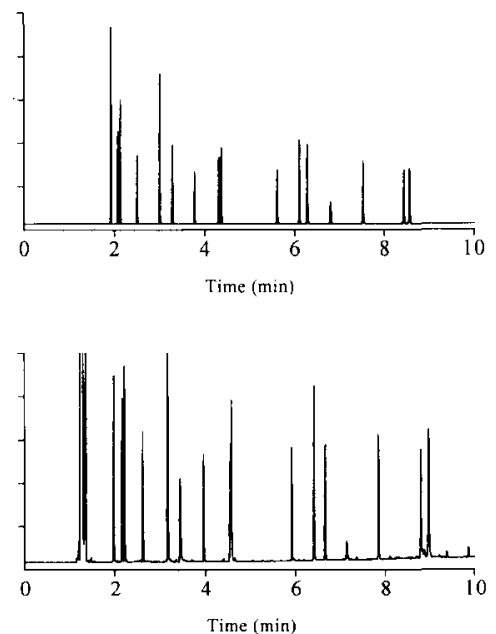

Column B
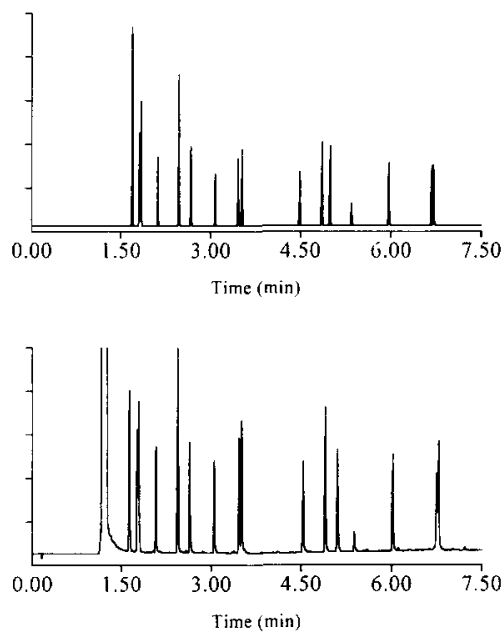

Column D
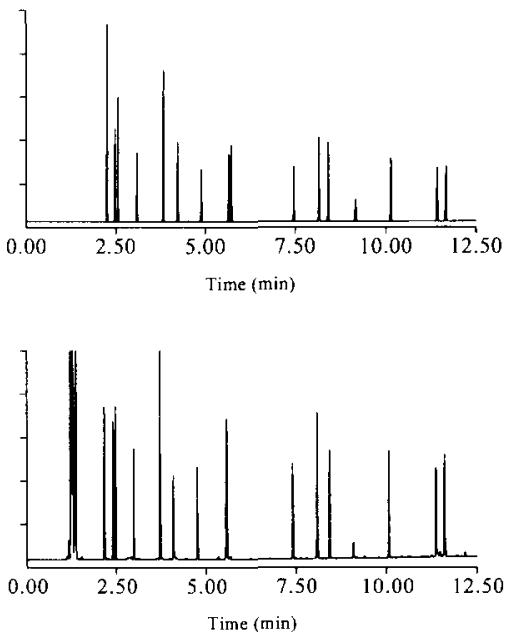

Fig. 4. Comparison of predicted (upper trace) and experimental (lower trace) chromatograms for multi-ramp temperature-programmed optimizations of the separation of the test mixture for all four the columns. The temperature programs located by the optimization are for column $\mathrm{A}: 105^{\circ} \mathrm{C} \rightarrow 13.8^{\circ} \mathrm{C} \mathrm{min}^{\prime} \rightarrow 242^{\circ} \mathrm{C} \rightarrow 23.6^{\circ} \mathrm{C} \mathrm{min}^{-1} \rightarrow 300^{\circ} \mathrm{C}$; column B: $91^{\circ} \mathrm{C} \rightarrow 24.8^{\circ} \mathrm{C}$ min ${ }^{1} \rightarrow 245^{\circ} \mathrm{C} \rightarrow 21.4^{\circ} \mathrm{C}^{\circ} \min ^{1} \rightarrow$ $300^{\circ} \mathrm{C}$; column $\mathrm{C}: 99^{\circ} \mathrm{C} \rightarrow 19.3^{\circ} \mathrm{C}$ min ${ }^{1} \rightarrow 243^{\circ} \mathrm{C} \rightarrow 19.9^{\circ} \mathrm{C}_{\text {min }}^{-1} \rightarrow 300^{\circ} \mathrm{C}$; column $\mathrm{D}: 105^{\circ} \mathrm{C} \rightarrow 13.8^{\circ} \mathrm{C}$ min ${ }^{-1} \rightarrow 266^{\circ} \mathrm{C} \rightarrow 15.0^{\circ} \mathrm{C}$ $\min ^{-1} \rightarrow 300^{\circ} \mathrm{C}$.

on column A, C and D again reveal partial peak overlap for the second critical peak pair. Again, additional peak broadening due to injection is the most probable cause for these observations. The data further indicate that for separation of the test mixture column B should be used under the multi-ramp temperature-programmed conditions indicated in Fig. 4.

It should be noted that, in principal, more variables could be added to the optimization for further 
optimization of the separation. Moreover, the optimization could be extended to include other column lengths and/or phase ratios.

\section{Conclusions}

With the optimization approach presented here it is possible to simulate and optimize both single- and multi-ramp temperature-programmed GC separations. Moreover, the approach allows selection of the capillary column most suited for the separation problem under study. The most attractive feature of the work presented here is that the optimizations can be performed without any experimental effort. This assures truly off-line optimizations and column selections

\section{References}

[1] H. Snijders, H.-G. Janssen and C. Cramers, J. Chromatogr. A, 718 (1995) 339.

[2] W. Wegscheider. E.P. Lankmayr and M. Otto, Anal. Chim. Acta, 150 (1983) 87.

[3] H.J.G. Debets, B.L. Bajema and D.A. Doornbos, Anal. Chim. Acta, 151 (1983) 131.

[4] P.J. Schoenmakers (Editor), Optimization of Chromatographic Selectivity, Elsevier, Amsterdam, 1986, Ch. 4.

[5] E. Dose, Anal. Chem., 59 (1987) 2420.

[6] J.A. Nelder and R. Mead, Computer J., 7 (1965) 308.

[7] S.L. Morgan and S.N. Deming, J. Chromatogr., 112 (1975) 267.

[8] P.A. Leclercq and C.A. Cramers, J. High Resolut. Chromatogr. Chromatogr. Commun., 8 (1985) 764. 